\section{Cahiers de Narratologie}

Analyse et théorie narratives

10.2 | 2001

La voix narrative

\title{
La voix narrative dans le roman dialogué : Diálogo en re mayor de Javier Tomeo
}

\section{Anne-Marie Vanderlynden}

\section{(2) OpenEdition}

\section{Journals}

Édition électronique

URL : http://journals.openedition.org/narratologie/10306

DOI : 10.4000/narratologie.10306

ISSN : 1765-307X

Éditeur

LIRCES

\section{Édition imprimée}

Date de publication : 1 janvier 2001

Pagination : 477-482

ISBN : 2914561032

ISSN : 0993-8516

\section{Référence électronique}

Anne-Marie Vanderlynden, « La voix narrative dans le roman dialogué : Diálogo en re mayor de Javier Tomeo ", Cahiers de Narratologie [En ligne], 10.2 | 2001, mis en ligne le 01 janvier 2001, consulté le 11 juin 2020. URL : http://journals.openedition.org/narratologie/10306 ; DOI : https://doi.org/10.4000/ narratologie.10306 


\title{
LA VOIX NARRATIVE DANS LE ROMAN DIALOGUÉ : \\ Diálogo en re mayor de Javier TOMEO
}

\author{
Anne-Marie VANDERLYNDEN \\ Université de Rouen
}

Comme son titre l'indique, ce roman est constitué par le récit d'une conversation entre deux interlocuteurs, Juan M. et Dagoberto. L'échange se déroule dans le compartiment d'un train omnibus qui ne transporte que ces deux passagers. Juan M. s'est vu assigner la place qui fait face à celle occupée par Dagoberto. Juan M., qui est aussi le narrateur, dit ne pas avoir cherché à s'installer ailleurs pour se soustraire à une éventuelle remarque du contrôleur, qui par ailleurs ne se manifestera pas pendant le voyage. Le texte est essentiellement constitué par les propos des co-énonciateurs, rapportés alternativement et dans des proportions quasiment identiques, au style direct et au style indirect. Le reste du contenu renvoie au dialogue de Juan M. avec lui-même et d'une certaine manière avec le lecteur (bien qu'il ne soit fait à celui-ci aucune place discursive), dans lequel il explique pourquoi il fait ce qu'il fait (le faire se limitant pratiquement, pour ce personnage à un dire, sauf pour ce qui est du dénouement), donne ses interprétations des propos et des gestes (au sens étroit et au sens large du terme puisque Juan M. est à l'affût de tous les indices pouvant le renseigner sur Dagoberto), à ses ressentis et à quelques éléments descriptifs : la tombée du jour, les gares, un feu dans la campagne, etc. Les échanges sont ponctués par des silences. Echanges verbaux et silences sont fréquemment doublés par un dialogue visuel : celui des regards. Les différents aspects du bruit du train et le rire des prostituées dont le conducteur de la locomotive facilite le travail en prolongeant les arrêts du convoi en gare, constituent les éléments dans lesquels s'évanouissent tout ensemble l'effort réaliste et les différentes figures de mise en abyme. A aucun moment Juan M. ne dit ou ne laisse entendre pourquoi 
il rapporte cette conversation qui s'avère être une suite d'expériences cuisantes pour lui, tant sur le plan verbal (les plus nombreuses) que sur le plan factuel (les plus frappantes ?). A aucun moment Juan M. ne dit ou ne laisse entendre à qui il s'adresse. Vue l'image qu'il donne de lui comme initiateur et acteur du dialogue en question, le lecteur (ou tout au moins un certain type de lecteur) peut même penser qu'il n'aurait jamais dû divulguer semblable expérience, expérience qu'il s'impose à lui-même dès l'ouverture de la conversation, en imposant un dialogue à un partenaire qui n'en veut pas et qui le lui signifie sans grande ambiguïté dès « l'ouverture des hostilités » :

- Buenos días - le dije de buen talante -. Pero aquel hombre ni siquiera volvió el rostro para darme la bienvenida con la mirada. (p. 10)

A Juan M. que s'est présenté comme tromboniste, Dagoberto réplique :

- Los trombones de varas, concretamente - dijo - me han parecido siempre un recurso acústico de segundo orden. (p. 12)

Page 14, Juan M. rapporte :

Luego, sin que viniese a cuento, me confesó que sentía una aversión invencible por los hombres que se jactan de su propia importancia, sobre todo cuando esos hombres eran demasiado gordos :

- Me dan náuseas - dijo.

Le lecteur comprend donc clairement que pour Dagoberto, Juan M. est un importun qu'il cherche à décourager par l'insulte et la vexation, moyens dont la violence, c'està-dire l'inobservance maximale des principes de courtoisie et de coopération (principes dont on sait par ailleurs qu'ils sont difficiles à articuler pour la théorie pragmatique), masquent l'indirection formulatoire, laquelle, convenons-en, est minimale.

Ce qui est évident pour le lecteur, Juan M. ne le perçoit pas, bien qu'il le vive. Il est loisible de penser que l'action du roman se déroule sur une scène circonscrite par le point aveugle de la conscience de ce personnage. Notre narrateur 
homo/intradiégétique serait bridé, sur le plan superficiel de sa psyché, par ce qu'il croit être son caractère affable :

Yo era entonces un hombre extravertido (...) que se sentía bastante satisfecho de sí mismo y que confiaba en su magnetismo personal y en la buena disposición del prójimo para aceptar el diálogo (p. 10)

La situation de double enfermement (le compartiment du wagon et l'opiniâtreté de Juan M.) est scellée à partir du moment où Dagoberto énonce le contrat de parole qu'il propose à Juan M. et que ce dernier, compte tenu de ce qu'il est, ne peut qu'accepter :

Usted me ha ofrecido amablemente la oportunidad de conversar (...). De acuerdo, podemos conversar (...). Antes de continuar, (...) quisiera hacerle alguna precisión. (...)...me molesta en sumo grado que la gente con la que estoy obligado a compartir unos cuantos metros cuadrados trate de venderme sus ideas $i$ Me sigue usted? (p. 16)

(Dagoberto lui demande l'impossible : être autre chose que ce qu'il est.)

Respondí que sí (...). Y entonces (...) se definió como una especie de Robinson Crusoe que estaba buscando una isla en la que naufragar.

- Una isla solitaria -susurró. (p. 16)

Quoi qu'il en dise, en croie, Juan M. accepte un contrat qu'il ne comprend pas, au sens où il n'en saisit pas l'implication majeure, qui serait qu'il disparaisse, au moins verbalement, ou, dans le meilleur des cas, qu'il offre à Dagoberto un dialogue d'une qualité autre que celui qu'il a entamé, ce que son interlocuteur lui fait remarquer :

Voy a decirle sin rodeos qué es lo que ha estado diciendo usted desde que se metió en este departamento. Tonterías (...). Cometió la ingenuidad de pensar que el diálogo es algo fácil. (p. 23)

Le malentendu, par lequel se justifie l'existence du texte, réside dans le sens du mot « diálogo » et, au delà, dans la difficulté de superposer les valeurs référentielles d'un mot.

L'essentiel des «événements » rapportés par Juan M. sont des paroles, ainsi que leurs effets, qui n'agissent, au 
mieux, que dans les limites du wagon. La restriction de champ opérée par le choix de la première personne de narration et renforcée par l'absence de témoin donne les effets des propos de Juan M. sur Dagoberto (impatience, désintérêt, voies de faits, etc.) en focalisation externe et présente toute manifestation et toute particularité physique de celui-ci (yeux rougis, sourire, canine métallique, chaussettes de teintes différentes, etc.) comme autant de signes que Juan M. s'empresse d'interpréter (sa voix intérieure - qualifiée : «Con esas palabras, en apariencia inocentes, quiso tal vez darme a entender que mi voz resultaba también demasiado atiplada para un hombre de pelo en pecho. ", p. 83 - rivalisant avec sa voix proférée) en fonction de sa grille personnelle dont la partie implicite émerge rapidement : le principe dialogal sousjacent au principe déclaré (cf. supra, p. 10) est qu'il faut plaire à son interlocuteur, lui offrir ce que l'on pense qu'il vous demande de lui offrir, lui laisser la part belle pour que, vous en sachant gré, il vous montre (ou vous laisse penser) qu'il vous tient en estime et continue à vous écouter, c'est-à-dire vous laisse lui parler.

Juan M., qui a une place discursive nécessairement prépondérante, est donc amené à réduire progressivement le lieu d'inscription de sa personne aussi bien sur le plan de son encyclopédie (Dagoberto en sait plus que lui sur le trombone), sur le plan argumentatif (les nombreuses occurrences de la séquence "reconocí que »), que sur le plan de la dignité personnelle que l'arsenal de sa stratégie d'évitement est impuissant à sauver de l'état de peau de chagrin. Un dégonflement symbolique signifié par la diminution de l'espace qu'il occupe dans le compartiment - et pourtant c'est un homme de bonne taille, cf. supra p. 14, cf., de même p. 56 " obesidad de panadero »- puisqu'il se voit confisquer son pantalon, son existence affective (la photo de Graciela), sa valise et ses partitions et qu'il doit se réfugier dans les toilettes (image du cul de sac dans lequel il s'est fourré). Heureusement (et malheureusement) il voyage sans son trombone. Son entêtement à vouloir converser contre la volonté de son interlocuteur le conduit à accepter un simulacre de dialogue qu'il accepte de le recommencer autant de fois qu'il plaît à Dagoberto puis de se taire avant de sauter en marche après 
avoir toutefois accepté de débarrasser Dagoberto de la vie en utilisant l'arme de celui-ci, un pistolet chargé avec une langue de belle-mère.

Dans quelles conditions peut-on exister par la parole, quel prix faut-il payer pour cela, qui faut-il être et pour quel interlocuteur, voilà la leçon que semble nous donner Juan M., leçon qu'il est incapable de tirer lui-même puisque les termes de son récit rétrospectif, dont la retrospectivité s'efface dès que le dialogue est rapporté au style direct (et alors le lecteur devient une sorte de témoin sans grands moyens) - effacement qui peut signifier que Juan M. pourrait revivre exactement la même expérience - marquent qu'il est incapable de prendre la moindre distance (à l'exception du flou de l'adverbe "entonces », p. 10, de la concession "..pero tampoco entonces comprendí muy bien el significado de aquellas palabras. », p. 106) par rapport à ce que l'on peut qualifier de « passion » (« Me levanté del asiento y extendí los brazos en cruz ». p. 138). L'incapacité à tirer leçon et à se changer soi-même est l'un des sens que l'on peut prêter à la dernière phrase du roman: "Lo que pasó después sin embargo ya no importa tanto.»(p. 143). Juan M. reste prisonnier du miroir :

«Seguramente no soy tan listo y simpático como pensaba », me dije asimismo.

Puede que fuese una tontería, pero aquella reflexión me sirvió de consuelo y me dio fuerzas para afrontar la última parte del viaje con más resignación.(p. 110)

Seul reste donc le narratif, un narratif sans « moralité » pour l'esprit d'un individu qui s'est évertué à ne pas exister pendant son aventure et qui continue à ne pas exister comme narrateur (un " on » qu'un autre " on »-au sens étymologique : être doué de parole - a affublé du costume de la première personne) puisque son nom «Juan M. » lui et nous interdit l'illusion d'une identification précise aussi bien que celle d'un ancrage spatio-temporel précis : il va de la ville de $\mathrm{P}$ à la ville de $\mathrm{K}$, il a étudié au conservatoire de $\mathrm{L}$ et il préfère les montagnes du sud (de quoi ?).

Dans Diálogo en re mayor, Juan M., narrateur, locuteur, énonciateur et co-énonciateur (le terme d'Antoine Culioli a-t- 
il jamais trouvé de meilleure illustration ?), malgré son omniprésence, feint d'émettre un texte qui pourrait être celui d'un greffier anonyme, dactylographié pour on ne sait qui ; une sorte de procès verbal auquel un autre " on » ne peut prêter que la voix de ses yeux; une voix tue prêtée à une voix tuée, un « on » qui a malgré tout le droit de se demander pourquoi est racontée une histoire-dialogue qui n'aurait pas dû avoir lieu.

La voix (= modalité) narrative choisie repose entièrement sur le choix d'une voix (qualifiée par Dagoberto, cf. supra, p. 83) amuïe par le passage à l'écrit engagée dans la voie d'un dialogue qui est un dialogue de sourds. De quoi rester muet.

Mon édition de référence : Anagrama, Barcelone, 1998. 